\title{
Espessamento das raízes aéreas de espécies de Pandanus Parkinson (Pandanaceae) $^{1}$
}

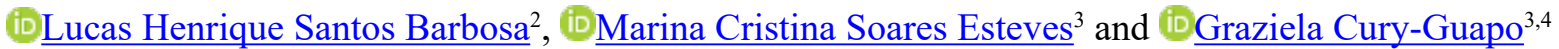

How to cite: Barbosa, L.H.S., Esteves, M.C.S., \& Cury-Guapo, G. 2021. Espessamento das raízes aéreas de espécies de Pandanus Parkinson (Pandanaceae) ${ }^{1}$. Hoehnea 48: e1062020. https://doi.org/10.1590/2236-8906-106/2020

\begin{abstract}
Thickening of aerial roots of Pandanus Parkinson (Pandanaceae) species) Monocotyledon roots and stems are organs that usually do not show secondary growth or thickness. However, it can occur and for the roots, the literature does not provide much information. Species of the genus Pandanus are easily found in urban areas and are characterized by the presence of thickened aerial roots, originating from the stem. To verify how thickening occurs, roots of $P$. utilis Bory, $P$. baptisti Hort., $P$. amaryllifolius Roxb e $P$. veitchii Hort samples of the roots were collected in urban areas, fixed and processed according to the usual technique for analysis under a light microscope. For all species, thickening occurs in the acropetal direction, through the formation and expansion of spaces of intercellular spaces in the cortex and in the vascular cylinder, meristematic activity of the proendodermis, division and increase in volume of the parenchymatic cells of the cortex and the vascular cylinder and meristematic activity of the pericycle.
\end{abstract}

Keywords: endodermis, monocotyledonous, pericycle, plant anatomy

RESUMO - (Espessamento das raízes aéreas de espécies de Pandanus Parkinson (Pandanaceae)). Raízes e caules de monocotiledôneas são órgãos que, usualmente, não apresentam crescimento secundário ou em espessura. Porém, pode ocorrer e, para as raízes, a literatura não fornece muitas informações. Espécies do gênero Pandanus são facilmente encontradas em áreas urbanas e se caracterizam pela presença de raízes aéreas espessadas, oriundas do caule. Para verificar como ocorre o espessamento, raízes de P. utilis Bory, P. baptisti Hort., P. amaryllifolius Roxb e P. veitchii Hort, foram coletadas amostras das raízes em áreas urbanas, fixadas e processadas conforme técnica usual para análise ao microscópio de luz. Para todas as espécies o espessamento ocorre no sentido acrópeto, por meio da formação e expansão de espaços de espaços intercelulares no córtex e no cilindro vascular, atividade meristemática da proendoderme, divisão e aumento de volume das células parenquimáticas do córtex e do cilindro vascular e atividade meristemática do periciclo.

Palavras-chave: anatomia vegetal, endoderme, monocotiledôneas, periciclo

\section{Introdução}

O crescimento secundário ou em espessura nas plantas, ocorre majoritariamente naquelas com sementes no entanto, entre as espécies de monocotiledôneas, esse evento não é tão comum (Tomlinson \& Zimmermann 1969, Gifford \& Foster 1989). Embora algumas espécies tenham desenvolvido um meristema lateral, todas carecem de um câmbio vascular típico. Acredita-se que as monocotiledôneas perderam a capacidade de crescimento secundário quando perderam suas células procambiais (entre os tecidos do xilema e do floema) na mudança de feixes vasculares primários para uma anatomia fechada (Ragni \& Greb 2018).

No entanto, apesar de não apresentarem crescimento secundário, proveniente da ação de um câmbio, várias monocotiledôneas apresentam caules e raízes espessados e, em relação ao caule, muitos trabalhos já foram realizados com o intuito de descobrir como tal espessamento é possível com a ausência de um câmbio. Esse tema tem sido alvo de estudos e discussões entre pesquisadores desde o século XIX, até que Ball (1941) sugeriu o termo Meristema de Espessamento Primário - MEP (Primary Thickening Meristem - PTM) que foi adotado e consagrado até os dias atuais.

No entanto, Menezes et al. (2005) propuseram, por meio de consistentes bases anatômicas e teóricas, uma nova interpretação sobre o assunto. As autoras afirmaram que, de fato, esse MEP, nada mais é do que o periciclo que produz tecidos vasculares para o interior do órgão; ou o periciclo, juntamente com a endoderme, que produz células parenquimáticas radialmente dispostas, formando parte do córtex interno como ocorre em raízes, fato já registrado por Van Fleet (1961)

1. Parte do Trabalho de Conclusão de Curso do primeiro Autor

2. Universidade de São Paulo, Escola Superior de Agricultura Luiz de Queiroz, Departamento de Ciências Biológicas, Avenida Pádua Dias, 11, Vila Independência, 13418-900 Piracicaba, SP, Brasil

3. Universidade Federal de Alagoas, Instituto de Ciências Biológicas e da Saúde, Avenida Lourival Melo Mota, s/n, Tabuleiro dos Martins, 57072-900 Maceió, AL, Brasil

4. Autor para correspondência: grazielacury@hotmail.com 
No que se refere às raízes de monocotiledôneas, muitos estudos (Riopel \& Steeves 1964, Tomlinson 1969, Clarck \& Harris 1981, Wilder 1986, Scatena \& Menezes 1996, Alquini \& Morretes 1996, Seubert 1997, Vianna et al. 2001, Pita \& Menezes 2002, Rodrigues \& Estelita 2004) foram realizados com o intuito de verificar o processo de diferenciação celular em diferentes estágios de desenvolvimento, porém sem o objetivo de verificar qualquer característica que possa identificar um crescimento secundário.

Porém, raízes espessadas de monocotiledôneas também já foram analisadas e o primeiro registro encontrado na literatura é um trabalho com Dracaena hookeriana K.Koch (Dracenaceae), no qual é relatado que o seu crescimento secundário é decorrente das divisões celulares do periciclo (Cheadle 1937).

Em raízes de Dioscorea sp. (Dioscoraceae), bem como em Asparagales, também foi registrada a ocorrência de um espessamento secundário desenvolvido por um "câmbio" que produz feixes vasculares secundários em meio às células do tecido fundamental (Tomlinson \& Zimmerman 1969, Carlquist 2012, Raman et al. 2014, Tomescu \& Groover 2019). Entretanto, não foi possível estabelecer um padrão de como realmente esse espessamento ocorre, devido à falta de estudos nessa esfera do conhecimento. Já, em relação aos caules de monocotiledôneas, apesar de ainda cercar uma polêmica sobre a nomenclatura dos tecidos envolvidos, o processo é bem compreendido e parece ser uniforme entre as plantas.

Raízes espessadas são facilmente encontradas em espécies do gênero Pandanus Parkinson (Pandanaceae). São aéreas, adventícias, formadas a partir do caule e apresentamse em diversos graus de espessamento e comprimentos. Devido ao aspecto distinto que essas raízes conferem às plantas, além de outros atributos inerentes a elas, são bastante utilizadas em projetos de paisagismo e arborização urbana devido ao seu potencial ornamental.

A família à qual o gênero em questão pertence, Pandanaceae, está inserida no grupo das monocotiledôneas, na ordem Pandanales que possui cinco famílias. Dentre elas, Velloziaceae, Triuridaceae e Cyclataceae, possuem representantes nativos do Brasil (Souza \& Lorenzi 2012), porém Pandanaceae não. Fósseis já foram encontrados na região Indo-Malaia-Melanésia (Stone 1972), o que pode sugerir sua origem nessa região e os principais locais onde a família é encontrada com riqueza de espécies são Nova Guiné, Austrália, Filipinas, Bornéu, Sudeste da Ásia e Ilha de Madagascar (Stone 1982). É composta por plantas arborescentes, lenhosas ou lianas, incluindo cerca de 700 espécies distribuídas em cinco gêneros, Benstonea, Freycinetia, Martellidendron, Pandanus e Sararanga, dos quais Pandanus é o mais diverso da família, com cerca de 450 espécies (Callmander et al. 2013).

Devido à grande lacuna científica relacionada ao espessamento de raízes de monocotiledôneas e com o intuito de contribuir para o esclarecimento desse evento, este trabalho investigou os mecanismos e os tecidos envolvidos no processo de espessamento em raízes de quatro espécies de Pandanus.

\section{Material e métodos}

Agrande ocorrência de plantas de espécies de Pandanus Parkinson em ambientes urbanos, a facilidade de coleta, a grande quantidade de raízes que possuem diversidade de espessuras e comprimentos e a uniformidade nos solos e ambientes das coletas são características que fizeram das espécies aqui estudadas, serem um bom modelo para analisar o espessamento em raízes de monocotiledôneas.

A coleta das amostras teve como critério obter material de diferentes distâncias a partir do ápice radicular $(3,5$, 7, 10, 12 e $15 \mathrm{~cm}$ de distância), o que foi suficiente para atingir diversidade nos graus de espessamento para todas as espécies analisadas (Pandanus utilis Bory (figura $1 \mathrm{a}$ ), P. baptisti Hort. (figura 1 b), P. amaryllifolius Roxb. (figura 1 c) e $P$. veitchii Hort. (figura $1 \mathrm{~d}$ ).

A coleta foi realizada, com o uso de um mini serrote, a partir de quatro indivíduos para cada espécie, em áreas urbanas da cidade de Maceió, Estado de Alagoas, Brasil, encontradas em solo seco, o que coloca todas as plantas coletadas nas mesmas condições de crescimento. Após a coleta, as amostras de raízes foram fixadas em FAA (formaldeído, ácido acético, etanol 70\%, 1:1:18 v/v) (Johansen 1940) por 24 horas e então armazenadas em etanol 70\%. Para as análises morfológicas, as amostras foram fotografadas, medidas e os seus detalhes foram observados em estereomicroscópio. Para as análises anatômicas, foram realizados cortes à mão com lâminas de barbear, nos planos transversal e longitudinal, dos fragmentos das raízes nas porções já mencionadas acima. Os cortes foram clarificados com hipoclorito de sódio a $2 \%$ e corados com solução aquosa de azul de Astra $0,5 \%$ e safranina $0,5 \%$ (Bukatsch 1972) ou com azul de toluidina (O’Brien et al.1965) e montados entre lâmina e lamínula com glicerina a $40 \%$ para a confecção de lâminas histológicas semi-permanentes. Também foram realizados testes com lugol (Johansen 1940) para detecção de amido.

Após a obtenção das lâminas histológicas procedeuse suas análises e captura de imagens em Microscópio Olympus BX51, com câmera acoplada Olympus DP25, software Olympus DP2-BSW. Foram coletadas também folhas para a confecção de exsicatas que foram depositadas no Herbário MHN (Museu de História Natural da Universidade Federal de Alagoas), sob os números de registro 4539 (P. utilis), 4540 (P. amaryllifolius), 4541 ( $P$. veitchii) e 4542 ( $P$. baptisti). Os parâmetros anatômicos analisados e registrados foram apenas aqueles relacionados diretamente com o espessamento da raiz.

\section{Resultados}

Análises morfológicas - Em Pandanus utilis Bory a emissão das raízes ocorre na porção inferior do caule (figura 1 a), enquanto nas demais espécies a emissão ocorre a partir da base das folhas (figuras 1 b-d). Nas quatro espécies, as raízes aéreas são emitidas com diferentes diâmetros e, conforme a raiz cresce em direção ao solo, o espessamento torna-se maior, no sentido acrópeto. Na região apical foi verificada uma coifa bem desenvolvida e, a partir de cerca de $10 \mathrm{~cm}$ acima do ápice e ao longo de todo o comprimento 

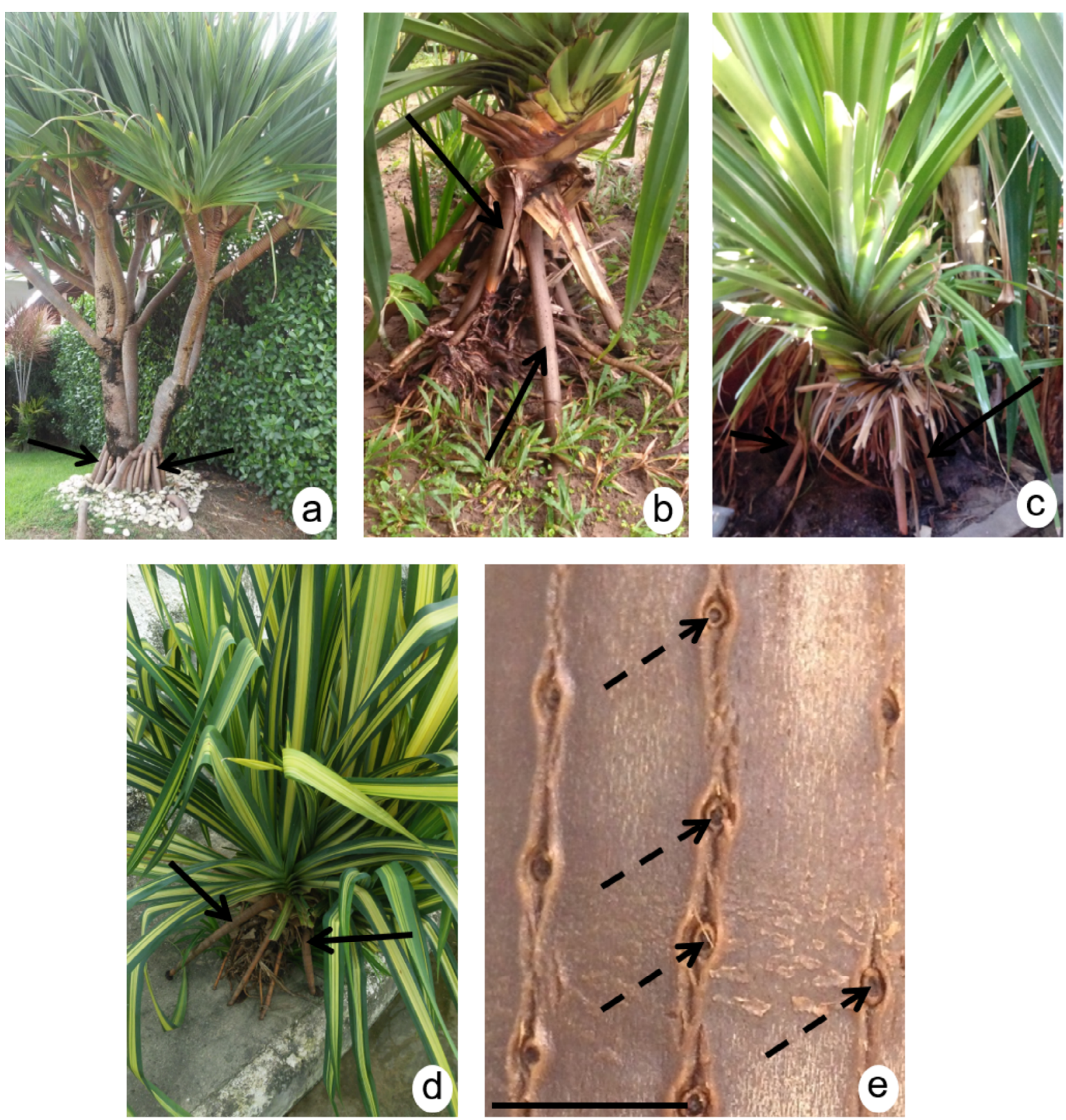

Figura 1. Espécies de Pandanus Parkinson no campo. a, e. P. utilis Bory. b. P. amaryllifolius Roxb. c. P. veitchii Hort. d. P. baptisti Hort. setas pretas: raízes adventícias aéreas; setas tracejadas: iniciação das raízes laterais. Barra $=3,0 \mathrm{~cm}$.

Figure 1. Pandanus species in the field. a, e. P. utilis Bory. b. P. amaryllifolius Roxb. c. P. veitchii Hort. d. P. baptisti Hort. black arrows: aerial adventitious roots; dashed arrow: initiation of lateral roots. $B a r=3,0 \mathrm{~cm}$.

das raízes, foram observadas pequenas saliências alinhadas (figura $1 \mathrm{e}$ ), as quais correspondem, de acordo com as análises anatômicas, à iniciação de raízes laterais, que só se desenvolvem após a penetração no solo, levando à sua ramificação.

Análises anatômicas - Em todas as espécies, nas regiões próximas ao ápice, o córtex era composto por meristema fundamental e já com alguns espaços intercelulares, formados por processo esquizógeno (figura 2). Algumas células do meristema fundamental em $P$. amaryllifolius Roxb. continham amido (figura 3 a) e em P. utilis idioblastos cristalíferos contendo ráfides (figura $3 \mathrm{~b}$ ). Também foi observado o córtex interno formado por várias camadas de células estratificadas nas quatro espécies, provenientes de divisões periclinais da proendoderme (camada precursora da endoderme) e suas derivadas (figuras $3 \mathrm{~d}-\mathrm{f}$ ).

Os tecidos condutores, bem como todas as outras células do cilindro vascular, nos primeiros estágios de desenvolvimento apresentavam todas as suas células derivadas ainda não diferenciadas e o restante do cilindro vascular já com espaços intercelulares de contorno arredondado em todas as espécies, sempre formados como os do córtex e delimitados por células parenquimáticas (figuras $3 \mathrm{c}-\mathrm{f}$ ). $\mathrm{O}$ periciclo era constituído por uma camada celular em P. utilis (figura 4 a), P. baptisti Hort. (figura 4 b) e em P. amaryllifolius (figura $4 \mathrm{c}$ ) e duas em P. veitchii Hort. (figura $4 \mathrm{~d}$ ). 

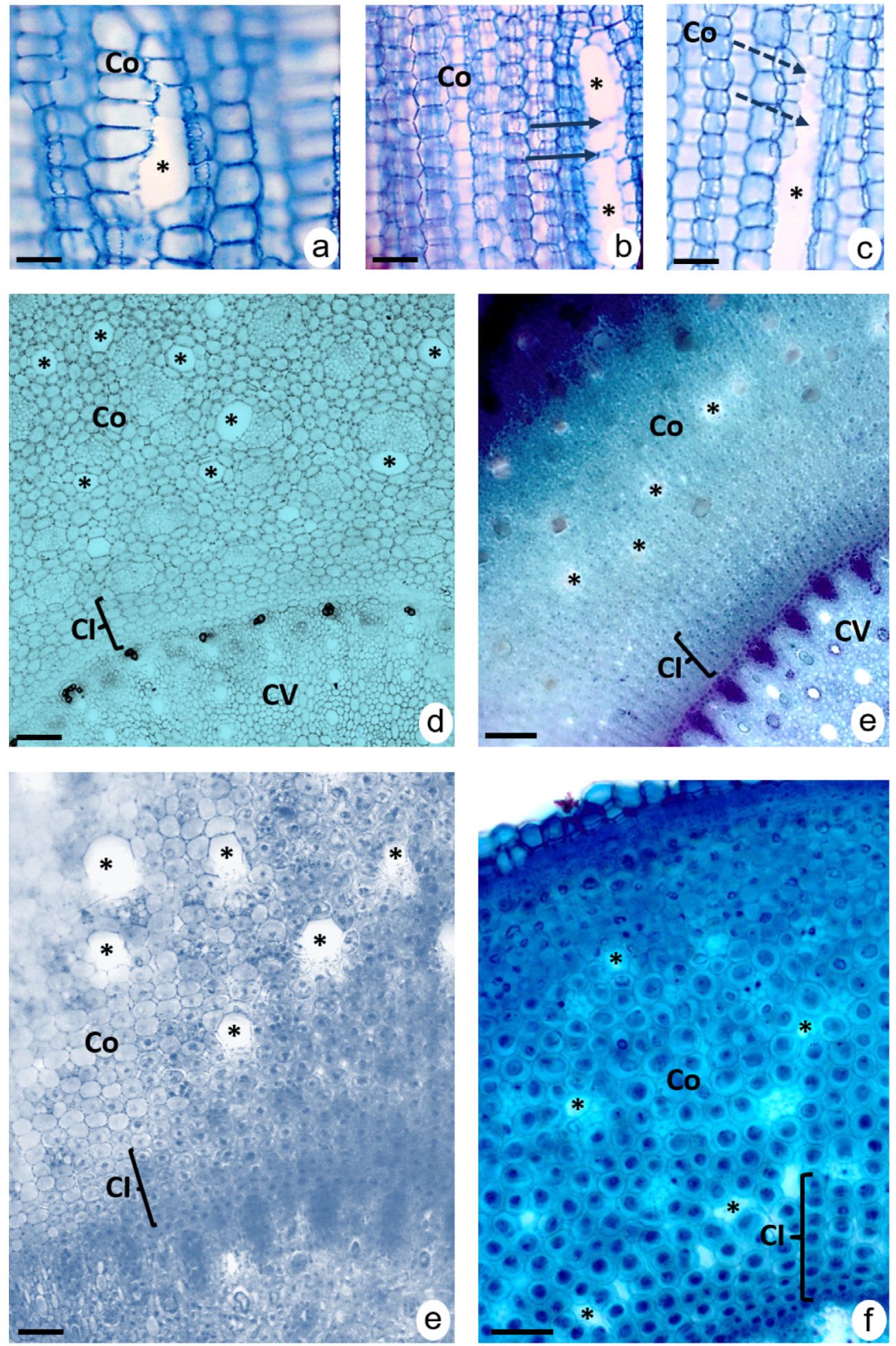

Figura 2. Espécies de Pandanus Parkinson. a. Corte longitudinal de P. utilis Bory. b. Corte longitudinal de $P$. amaryllifolius Roxb. c. Corte longitudinal de P. veitchii Hort. d. Corte transversal de $P$. utilis. e. Corte transversal de P. amaryllifolius. f. Corte transversal de $P$. veitchii. g. Corte transversal de $P$. baptisti Hort. Co: córtex; CI: córtex interno; CV: cilindro vascular; *: espaços intercelulares; setas azuis: septos entre os espaços intercelulares; setas azuis tracejadas: locais de esquizogênese celular. Barras $=50 \mu \mathrm{m}$ (a, f, g); $100 \mu \mathrm{m}(\mathrm{b}, \mathrm{c}, \mathrm{d}, \mathrm{e})$.

Figure 2. a. Pandanus species Parkinson. P. utilis Bory longitudinal sections. b. P. amaryllifolius Roxb. longitudinal sections. c. P. veitchii Hort. longitudinal sections. d. P. utilis crossections. e. P. amaryllifolius crossections. f. P. veitchii crossections. g. P. baptisti crossections. Co: cortex; CI: inner cortex; CV: vascular cylinder; *: intercellular spaces; blue arrows: septa between the vascular spaces; blue dashed arrows: cell schizogenesis site. Bars $=50 \mu \mathrm{m}(\mathrm{a}, \mathrm{f}, \mathrm{g}) ; 100 \mu \mathrm{m}(\mathrm{b}, \mathrm{c}, \mathrm{d}, \mathrm{e})$. 

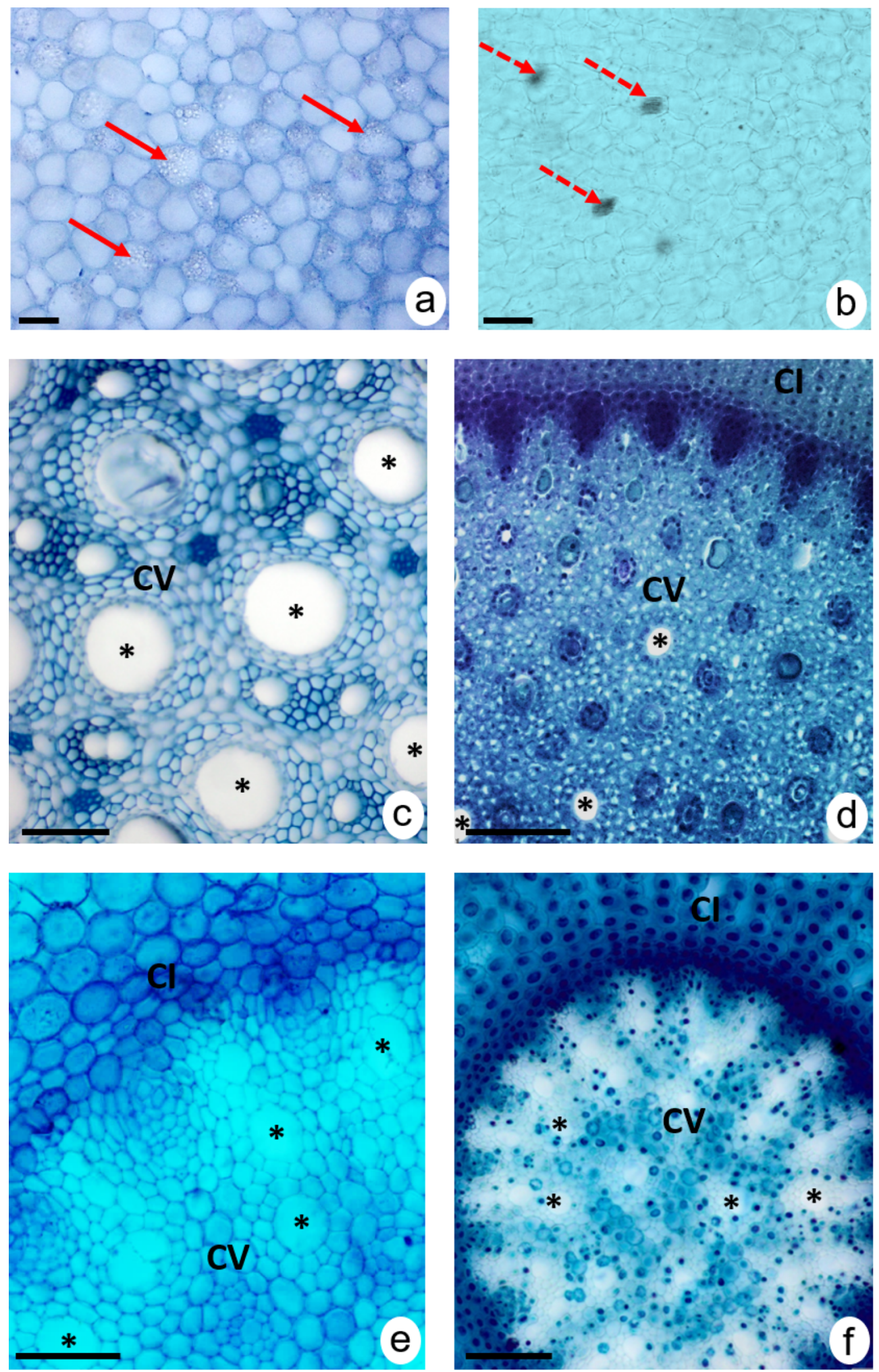

Figura 3. Cortes transversais das raízes de Pandanus Parkinson. b,c. P. utilis Bory. a,d. P. amaryllifolius Roxb. e. P. veitchii Hort. f. P. baptisti Hort. CI: córtex interno; CV: cilindro vascular; setas vermelhas: amido; setas vermelhas tracejadas: ráfides; *: espaços intercelulares. Barras $=50 \mu \mathrm{m}(\mathrm{a}, \mathrm{b}, \mathrm{f}) ; 100 \mu \mathrm{m}(\mathrm{c}, \mathrm{d}, \mathrm{e})$. Figure 3. Pandanus Parkinson roots crossections. a,c. P. utilis Bory. b,d. P. amaryllifolius Roxb. e. P. veitchii. f. P. baptisti. red arrows: starch; red dashed arrows: crystals; *: intercellular spaces. Bars $=50$ $\mu \mathrm{m}(\mathrm{a}, \mathrm{b}, \mathrm{f}) ; 100 \mu \mathrm{m}(\mathrm{c}, \mathrm{d}, \mathrm{e})$. 

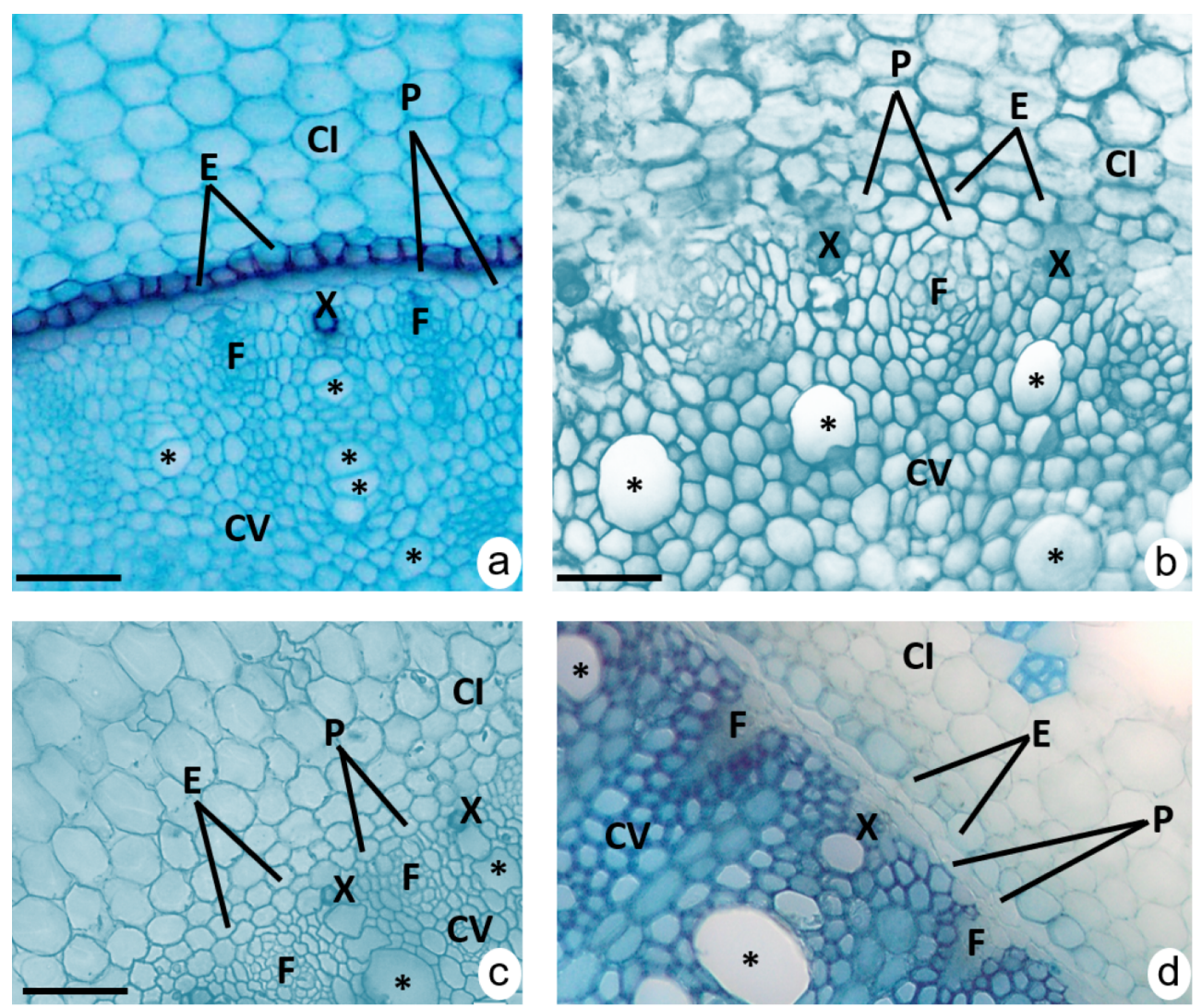

Figura 4. Cortes transversais das raízes de Pandanus Parkinson. a. P. utilis Bory. b. P. amaryllifolius Roxb. c. P. veitchii Hort. d. P. baptisti Hort. CI: córtex interno; CV: cilindro vascular; E: endoderme; F: floema; P: periciclo; X: xilema. *: espaços intercelulares. Barras $=50 \mu \mathrm{m}$ (c); $100 \mu \mathrm{m}$ (a, b, d).

Figure 4. Pandanus Parkinson roots crossections. a. P. utilis Bory. b. P. amaryllifolius Roxb. c. P. veitchii Hort. d. P. baptisti Hort. CI: inner cortex; CV: vascular cylinder; E: endodermis; F: phloem; P: pericycle; $\mathrm{X}$ : xylem. *: intercellular spaces. Bars $=50 \mu \mathrm{m}(\mathrm{c}) ; 100 \mu \mathrm{m}(\mathrm{a}, \mathrm{b}, \mathrm{d})$.

Ao longo do desenvolvimento da raiz, os espaços intercelulares em $P$. utilis, apresentavam contorno arredondado tanto no córtex (figura 5 a), quanto no cilindro vascular (figura $5 \mathrm{~b}$ ); P. amaryllifolius (figura $5 \mathrm{c}$ ), $P$. veitchii (figura 5 e) e $P$. baptisti (figura $5 \mathrm{~g}$ ) apresentavam espaços de contorno longitudinal a irregular no córtex e, no cilindro vascular, contorno arredondado (figuras $5 \mathrm{~d}, \mathrm{f}, \mathrm{h}$ ). Em plano longitudinal os espaços eram alongados (figuras 6 a-d) e em P. baptisti, no seu interior, foram encontradas astroesclereídes (figuras $6 \mathrm{e}, \mathrm{f}$ ).

Em níveis subsequentes, em todas as espécies, conforme a raiz seguia seu espessamento lateral, os espaços intercelulares continuaram a ser formados por processo esquizógeno e os espaços já estabelecidos anteriormente passaram a sofrer lise, aglutinando-se uns aos outros e tornando-se maiores, passando a ter contornos irregulares, como em P. baptisti (figuras 7 a-e).

O periciclo passou a sofrer divisões celulares (figuras $7 \mathrm{f}, \mathrm{g}, 8 \mathrm{a}-\mathrm{c}$ ), produzindo células parenquimáticas para $\mathrm{o}$ interior do cilindro vascular e constantemente "empurradas" centripetamente, devido ao contínuo processo de divisão do periciclo (figura $8 \mathrm{~d}$ ), que também forma as raízes laterais (figuras $8 \mathrm{e}, \mathrm{f}$ ).

Em todas as espécies estudadas, foi verificado que o espessamento da raiz ocorre pelos seguintes eventos: divisão e expansão das células parenquimáticas tanto no córtex, quanto no cilindro vascular; formação e expansão de espaços intercelulares, tanto no córtex, quanto no cilindro vascular; divisões celulares da camada precursora da endoderme, que produz a porção interna do córtex e; atividade meristemática do periciclo que produz células parenquimáticas para o interior do cilindro vascular. Essas células parenquimáticas são constantemente "empurradas" centripetamente, devido ao contínuo processo de divisão do periciclo.

\section{Discussão}

Em todas as espécies de Pandanus Parkinson aqui analisadas, foram observados espaços intercelulares que podem apresentar contorno regular arredondado ou irregular, dependendo da espécie e do nível de desenvolvimento do órgão. A formação desses espaços por separação, e/ou separação seguida de lise e desintegração celular, ocorre em muitas espécies, tanto em folhas, quanto em caules e raízes, geralmente constituindo um aerênquima e relacionados com ambientes de solos alagadiços (Cutler et al. 2008, Evert, 2013), sendo de importância fundamental para adaptação das plantas nesse tipo de habitat (Seago et al. 2005). 

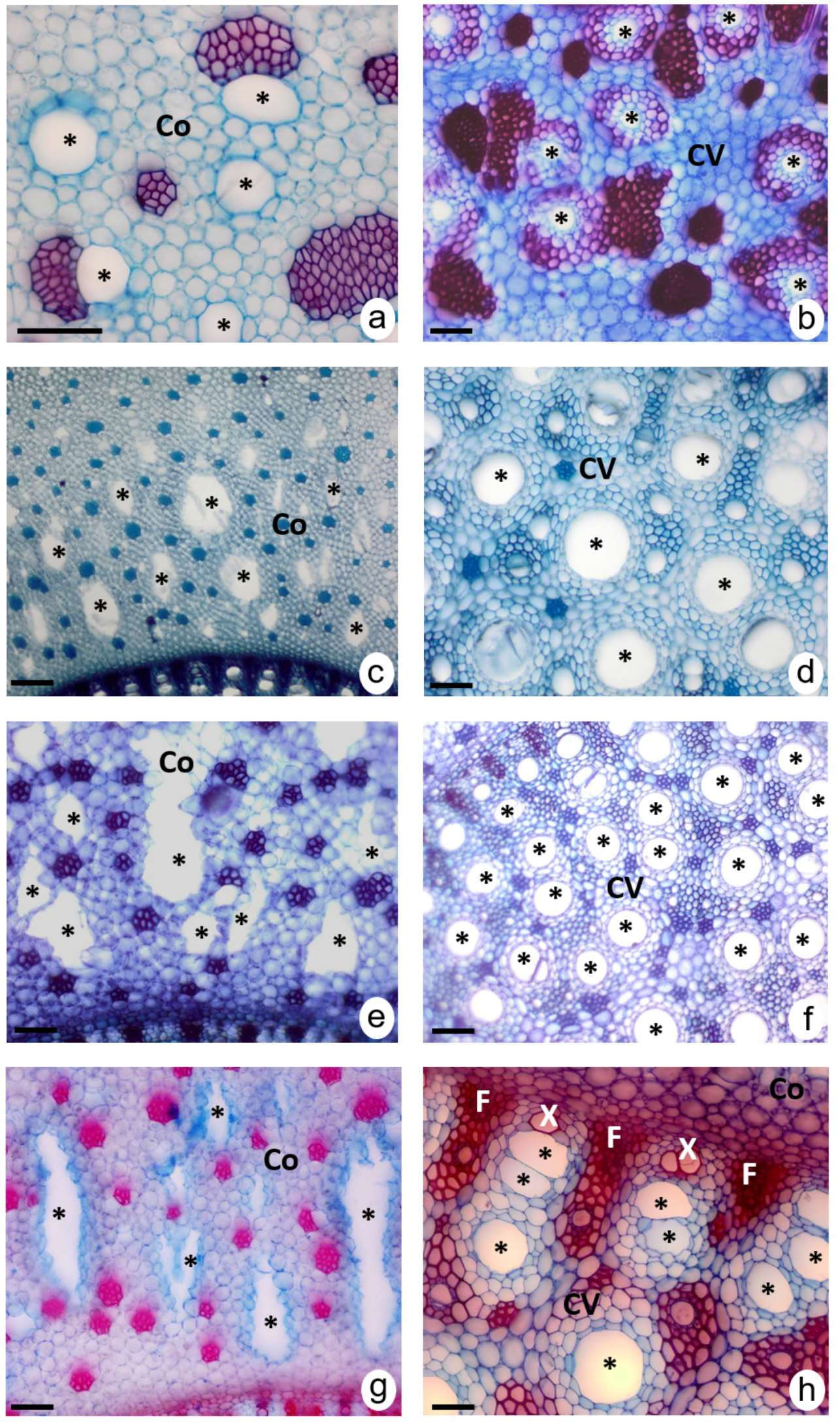

Figura 5. Cortes transversais das raízes de Pandanus Parkinson. a, b. P. utilis Bory. c, d. P. amaryllifolius Roxb. e, f. P. veitchii Hort. g, h. P. baptisti Hort. CI: córtex interno; Co: córtex; CV: cilindro vascular; F: floema; X: xilema. *: espaços intercelulares. Barras $=50 \mu \mathrm{m}(\mathrm{c}) ; 100 \mu \mathrm{m}(\mathrm{a}, \mathrm{b}, \mathrm{d})$.

Figure 5. Pandanus roots crossections. a, b. P. utilis Bory. c, d. P. amaryllifolius Roxb. e, f. P. veitchii Hort. g, h. P. baptisti Hort. CI: inner cortex; Co: cortex; CV: vascular cylinder; F: phloem; X: xylem. *: intercellular spaces. Bars $=50 \mu \mathrm{m}(\mathrm{c}) ; 100 \mu \mathrm{m}(\mathrm{a}, \mathrm{b}, \mathrm{d})$. 

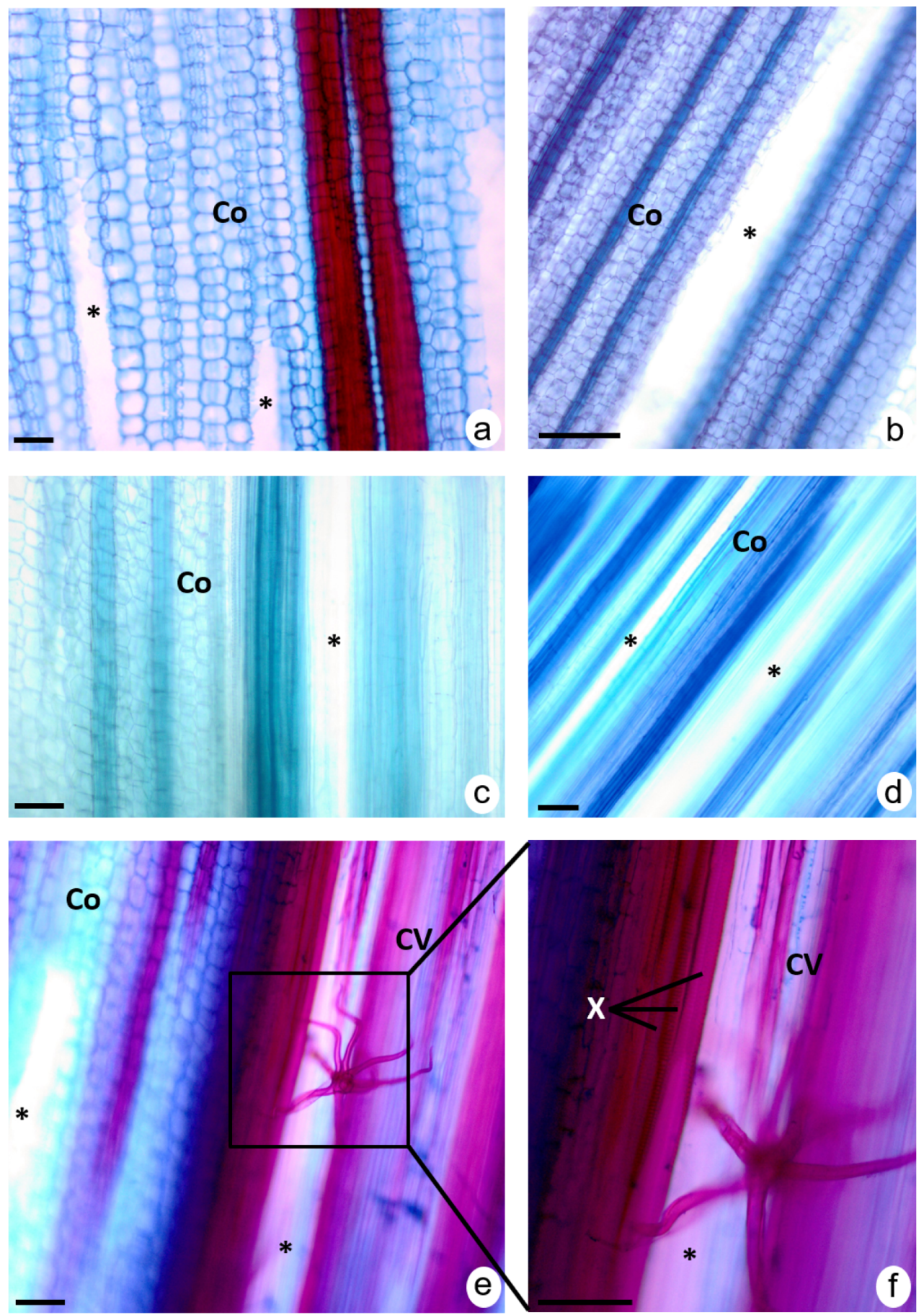

Figura 6. Cortes longitudinais das raízes de Pandanus Parkinson. a. P. utilis Bory. b. P. amaryllifolius Roxb. c. P. veitchii Hort. d. P. baptisti Hort. e. astroesclereíde em espaço intercelular de P. baptisti. f. detalhe da imagem anterior. Co: córtex; $\mathrm{CV}$ : cilindro vascular; $\mathrm{X}$ : xilema; *: espaços intercelulares. Barras $=50 \mu \mathrm{m}(\mathrm{a}-\mathrm{f})$.

Figure 6. Pandanus Parkinson longitudinal sections a. P. utilis Bory. b. P. amaryllifolius Roxb. c. P. veitchii Hort. d. P. baptisti Hort. e. astrosclereids in intercellular space of P. baptisti. f. detail from previous image. Co: cortex; CV: vascular cylinder; X: xylem; *: intercellular spaces. Bars $=50 \mu \mathrm{m}$ (a-f).

A questão aqui a ser esclarecida é como ocorre a presença de aerênquima em plantas coletadas em solo seco. Souza \& Lorenzi (2012) referem-se à Pandanaceae como uma família com distribuição predominantemente paleotropical, principalmente próximo às regiões litorâneas. Essas regiões geralmente possuem solo bastante úmido e, apesar das espécies aqui analisadas terem sido coletadas em solo seco, seu ambiente natural pode sofrer períodos de alagamento, o que justifica a presença de aerênquima como de fundamental importância para a sobrevivência dessas plantas. Porém, o que explica a presença de aerênquima nessas plantas, não é a presença ou ausência de água no solo, mas o fato de ser uma característica taxonômica do grupo determinada geneticamente e, tais mudanças, ao 

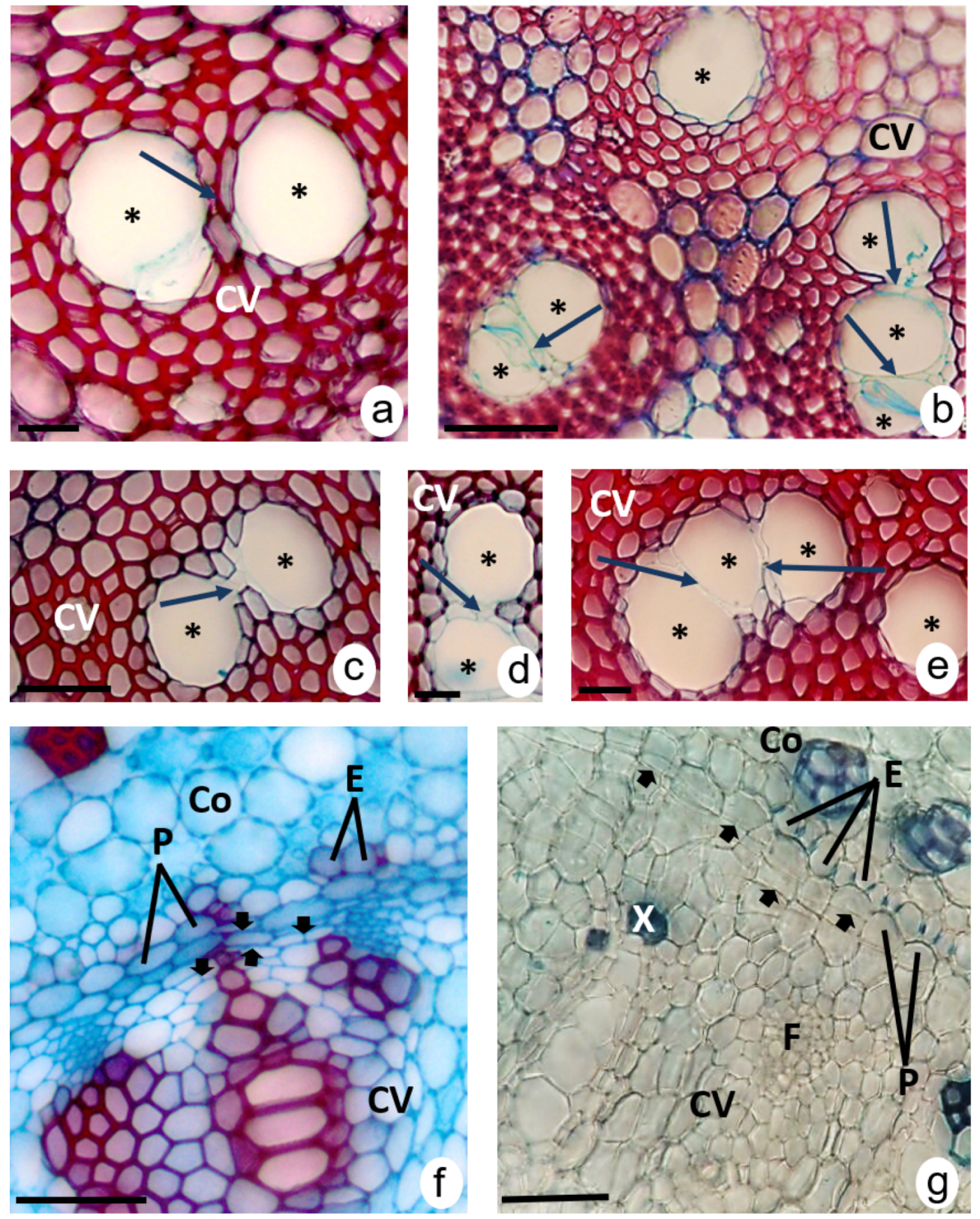

Figura 7. Cortes transversais das raízes de Pandanus Parkinson. a-e. P. baptisti Hort. f,g. P. utilis Bory. Co: córtex; CV: cilindro vascular; E: endoderme; F: floema; P: periciclo; X: xilema; setas azuis: septos entre os espaços intercelulares; *: espaços intercelulares; setas pretas pequenas: divisão de células do periciclo e de suas derivadas. Barras $=25 \mu \mathrm{m}$ (a, d, e); $50 \mu \mathrm{m}(\mathrm{b}, \mathrm{c}, \mathrm{f}) ; 200 \mu \mathrm{m}(\mathrm{g})$.

Figure 7. Pandanus Parkinson roots crossections. a-e. P. baptisti Hort. f,g. P. utilis Bory. Co: cortex; CV: vascular cylinder; E: endodermis; F: phloem; P: pericycle; X: xylem; blue arrows: septa between the vascular spaces; *: intercellular spaces; small black arrows: division of the pericycle and its derivatives cells. Bars $=25 \mu \mathrm{m}(\mathrm{a}, \mathrm{d}, \mathrm{e}) ; 50 \mu \mathrm{m}(\mathrm{b}, \mathrm{c}, \mathrm{f}) ; 200 \mu \mathrm{m}(\mathrm{g})$.

nível dos genes, estão menos sujeitas a homoplasias (Judd et al. 2009).

Entretanto, muitas outras pesquisas trazem evidências sobre a formação de aerênquima relacionada a fatores ambientais em plantas em solos secos, como no caso de indução por anaerobiose do solo (Peterson 1992, Jackson \& Armstrong 1999). Para Mani (1962), além da falta de oxigênio, a inanição também pode estar envolvida na formação dos espaços intercelulares lisígenos que podem ser decorrentes da alta lignificação de células corticais mais internas. Ademais, em condições de estresse edáfico e hipóxia, a formação de espaços aumenta a captura de água sob seca, aumentando os recursos internos disponíveis para um enraizamento mais profundo (Lynch 2018).

Trabalhos com muitas espécies cultivadas demonstram que a formação do aerênquima no córtex da raiz ocorre por meio da morte celular programada. A conversão de células corticais vivas em espaço para armazenamento de ar permite o reinvestimento de recursos nutritivos em outros tecidos vegetais, incluindo pontas de raízes em crescimento, e reduz o custo metabólico de manutenção do córtex radicular. Portanto, a hipótese é que a formação desses espaços é 

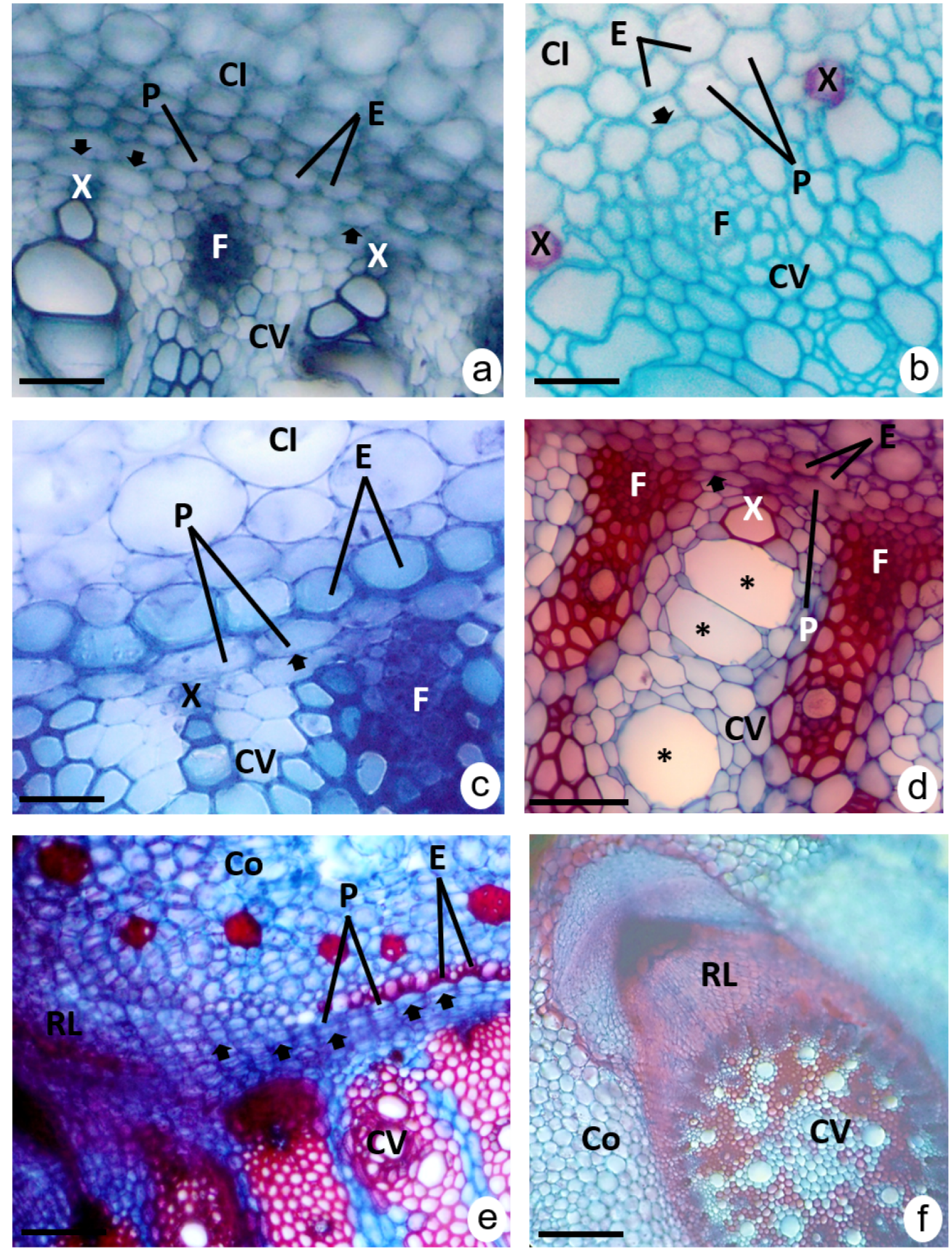

Figura 8. Cortes transversais das raízes de Pandanus Parkinson. a,e. P. utilis Bory. b. P. amaryllifolius Roxb. c, f. P. veitchii Hort. d. P. baptisti Hort. CI: córtex interno; Co: córtex; CV: cilindro vascular; E: endoderme; F: floema; P: periciclo; RL: raiz lateral; X: xilema; setas azuis: septos entre os espaços intercelulares; setas azuis tracejadas: locais de esquizogênese celular; setas pretas pequenas: divisão de células do periciclo e de suas derivadas. Barras $=25 \mu \mathrm{m}$ (c); $50 \mu \mathrm{m}$ (a, b, d); $200 \mu \mathrm{m}$ (e); $250 \mu \mathrm{m}$ (f).

Figure 8. Pandanus Parkinson roots crossections. a,e. P. utilis Bory. b. P. amaryllifolius Roxb. c, f. $P$. veitchii Hort. d. P. baptisti Hort. CI: inner cortex; Co: cortex; CV: vascular cylinder; E: endodermis; F: phloem; P: pericycle; RL: lateral root; X: xylem; blue arrows: septa between the vascular spaces; blue dashed arrows: cell schizogenesis site; small black cells: division of the pericycle and its derivatives cells. Bars $=25 \mu \mathrm{m}$ (c); $50 \mu \mathrm{m}$ (a, b, d); $200 \mu \mathrm{m}$ (e); $250 \mu \mathrm{m}$ (f).

vantajosa para a exploração e captação de recursos do solo sob estresse edáfico (Lynch \& Brown 1998, Fan et al. 2003). Além disso, condições subótimas de nitrogênio, fósforo, enxofre e água (Konings \& Verschuren 1980, Drew et al. 1989, Bouranis et al. 2003, Fan et al. 2003, Zhu et al. 2010, Postma \& Lynch 2011) podem estimular o aumento na produção de aerênquima cortical da raiz, o que permite que a raiz mantenha diâmetros maiores.

Seago et al. (2005) analisaram 85 espécies de plantas, dentre elas, cinco ordens de monocotiledôneas de locais úmidos, nas quais verificaram, a partir do desenvolvimento e organização de lacunas corticais, câmaras de ar ou 
cavidades em aerênquima ou espaços intercelulares. No entanto, a ausência de estudo em Pandanales, faz do presente trabalho, o primeiro registro para a ordem. Astroesclereídes foram observadas em alguns espaços intercelulares, células geralmente presentes em aerênquima de órgãos em ambientes aquáticos que mantém a integridade do tecido e facilita sua aeração, como ocorre no pecíolo de folhas de Thea e Nymphaea (Scatena \& Scremin-Dias 2012).

Nas regiões mais próximas do ápice, todas as espécies apresentaram estratificação conspícua das células do córtex interno, composto por células meristemáticas, derivadas das células meristemáticas precursoras da endoderme (proendoderme). No entanto, nas regiões diferenciadas a estratificação do córtex interno é pouco visível, devido ao espessamento do órgão com ocorrência de divisões celulares e ao aumento do volume dessas células (Menezes et al. 2005).

Neste trabalho, foi encontrado amido em células do meristema fundamental e a presença de amido nas células do meristema fundamental nas espécies estudadas fornece à planta a energia necessária para o seu crescimento e desenvolvimento (Hoffman 1999) e contribui com o processo de espessamento. Além disso, carboidratos também podem servir como fonte de energia em processos que antecedem a divisão celular (Kraus et al. 2004), o que poderia justificar sua localização apical, auxiliando inclusive no seu gravitropismo e no processo de ramificação abaixo do solo.

Os cristais, também encontrados nas espécies de Pandanus estudadas, são substâncias inorgânicas oriundas do metabolismo celular com importante defesa contra herbívoros, (Franceschi \& Horner 1980, Franceschi 2001, Nakata 2003, Corrêa et al. 2008, Munuswamy et al. 2016, Dardengo et al. 2017) e animais forrageiros causando irritação e sensação de queimação na boca (Kingsbury 1964). Além de defesa, esses cristais também podem estar relacionados a uma função excretora para as plantas que não possuem um sistema excretor para descartar restos sólidos como sais de cálcio (Paiva 2019).

Em relação às funções do periciclo em raízes de monocotiledôneas, a literatura, à exceção de Cheadle (1937), traz informações apenas para a produção de raízes aéreas laterais, como podemos observar nos trabalhos de Xiao et al. (2019), Seo et al. (2020) e TorresMartinez et al. (2019), não sendo encontradas no que diz respeito ao espessamento. A falta de literatura sobre a atuação do periciclo no espessamento de raízes de monocotiledôneas, provavelmente se deve ao fato de a maioria dos pesquisadores não aceitarem que o periciclo poderia participar desse espessamento, mas que um "câmbio", como nos caules de monocotiledôneas, por exemplo, seria o responsável por essa função.

Já atividade meristemática da endoderme, através das divisões celulares de sua camada precursora (proendoderme) para a produção de raízes laterais e para o espessamento das raízes, é tema mais comumente encontrado na literatura, ao contrário do periciclo. Isso ocorre devido à presença da endoderme em raízes, ser fato já há muito conhecido e aceito pelos pesquisadores (Menezes et al. 2005).

Os eventos relacionados com o processo de espessamento das raízes das espécies de Pandanus aqui analisadas envolvem os seguintes processos: divisões celulares do periciclo, o que até o momento para monocotiledôneas havia sido relatado apenas em caules (Van Fleet 1961, Menezes et al. 2005, Menezes et al. 2012); formação e expansão de espaços intercelulares (aerênquima); aumento de volume e divisão de células parenquimáticas, o que ainda não foi referido em outras espécies do gênero e; atividade meristemática da camada celular precursora da endoderme, fato já observado nas raízes de Canna edulis Kerr-Gawler (Alonso et al. 2004), Echinodorus panicullatus (Mart.) Buchenan, Cephalostemon riedelianus Koern, Cyperus papyrus L., Lagenocarpus junciformis (Kunth) O. Kunth, Lagenocarpus rigidus (Kunth) Nees e Zingiber officinale Roscoe (Menezes et al. 2005) e no gênero Scleria Berg (Lima \& Menezes 2008). Dentre esses eventos, dois deles, divisões celulares do periciclo e atividade meristemática da endoderme, também estão envolvidos no espessamento de caules de monocotiledôneas.

\section{Agradecimentos}

Agradecemos ao Prof. Dr. José Rubens Pirani do Instituto de Biociências da Universidade de São Paulo, por contribuir na identificação de algumas espécies: e ao Laboratório de Biologia Celular e Molecular do Instituto de Ciências Biológicas e da Saúde (UFAL), coordenado pelo Prof. Emiliano de Oliveira Barreto, por permitir a utilização do microscópio com sistema para análise e captura de imagens.

\section{Contribuição dos autores}

Lucas Henrique Santos Barbosa: conceito e design do estudo; coleta de dados; análise e interpretação dos dados; preparação do manuscrito.

Marina Cristina Soares Esteves: coleta de dados; análise de dados.

Graziela Cury-Guapo: conceito e design do estudo; coleta de dados; análise e interpretação dos dados; preparação do manuscrito; revisão e correção do manuscrito.

\section{Conflitos de interesse}

Não há conflitos de interesse.

\section{Literatura citada}

Alonso, A.A., Moraes-Dallaqua, M.A., Menezes, N.L. 2004. Endoderme com atividade meristemática em raiz de Canna edulis Kerr-Gawler (Cannaceae). Acta Botanica Brasílica, 18(3): 693-699.

Alquini, Y., Morretes B.L. 1996. Organização estrutural da raiz de Musa rosaceae Jacq. (Musaceae). Arquivos de Biologia e Tecnologia 39: 657-669. 
Ball, E. 1941. The Development of the Shoot Apex and of the Primary Thickening Meristem In: Phoenix canariensis Chaub., with Comparisons to Washingtonia filifera Wats. And Trachycarpus excelsa Wendl. American Journal of Botany 28: 820-832.

Bouranis DL, Chorianopoulou S, Siyiannis V, Protonotarios V, Hawkesford M. 2003. aerenchyma formation in roots of maize during sulphate starvation. Planta 217: 382-391.

Bukatsch, F. 1972. Bemerkungen zur Doppelfarbung: Astrablau-Safranin. Mikrokosmos 61: 255.

Callmander, M.W., Booth, T.J., Beentje, H., Buerki, S. 2013. Update on the systematics of Benstonea (Pandanaceae): When a visionary taxonomist foresees phylogenetic relationships. Phylotaxa, 112 (2): 57-60.

Carlquist, S. 2012. How wood evolves: a new synthesis Sherwin Botany 90: 901-940.

Cheadle, V.I. 1937. Secondary Growth by Means of a Thickening Ring in Certain Monocotyledons. Botanical Gazette 98 (3): 535-555.

Clarck, L.H., Harris, W.H. 1981. Observations on the root anatomy of rice (Oryzasativa L.). American Journal of Botany 68: 154-161.

Corrêa, PG., Pimentel, R.M.M., Cortez, J.S.A., Xavier, H.S. 2008. Herbivoria e anatomia foliar em plantas tropicais brasileiras. Ciência e Cultura 60: 54-57.

Cutler, D.F., Botha, T., Botha, C.E.J. \& Stevenson, D.W. 2008. Plant Anatomy: an Applied Approach. WileyBlackwell Press, Malden.

Dardengo, J.F.E., Rossi, A.A.B., Silva, I.V., Pessoa, M.J.G., Silva, C.J. 2017. Análise da influência luminosa nos aspectos anatômicos de folhas de Theobroma speciosum Willd ex Spreng. (Malvaceae). Ciência Florestal 27: 843-851.

Drew, M.C., He, C.J., Morgan, P.W. 1989. Decreased ethylene biosynthesis, and induction of aerenchyma, by nitrogen- or phosphate-starvation in adventitious roots of Zea mays L. Plant Physiology 91: 266-271.

Evert, R. 2013. Anatomia das Plantas de Esau - meristemas, células e tecidos do corpo a planta: sua estrutura, função e desenvolvimento. Blucher, São Paulo.

Fan, M.S., Zhu, J.M., Richards, C., Brown, K.M., Lynch, J.P. 2003. Physiological roles for aerenchyma in phosphorus-stressed roots. Functional Plant Biology 30: 493-506.

Franceschi, V.R., Horner, H.T. 1980. Calcium oxalate crystals in plants. The Botanical Review 46: 361-427.

Franceschi, V.R. 2001. Calcium oxalate in plants. Trends in Plant Science 6:1.

Gifford, E.M., Foster, A.S. 1989. Morphology and Evolution of Vascular Plants. Freeman, New York.

Hoffman, W.A. 1999. Fire and population dynamics of woody plants in a neotropical savanna: matrix model projections. Ecology 80:1354-1369.

Jackson, M.B., Armstrong, W. 1999. Formation of aerenchyma and the processes of plant ventilation in relation to soil flooding and submergence. Plant Biology 1: 274-287.
Johansen, D.A. 1940. Plant microtechnique. McGraw-Hill Book Company, New York.

Judd, W.S., Campbell, C.S., Kellogg, E.A., Stevens, P.F., Donoghue, M.J. 2009. Sistemática Vegetal: um Enfoque Filogenético. Artmed Editora, Porto Alegre.

Konings, H., Verschuren, G. 1980. Formation of aerenchyma in roots of Zea mays in aerated solutions, and its relation to nutrient supply. Physiologia Plantarum 49 (3): 265-270.

Kingsbury, J.M. 1964. Poisonous plants of the United States and Canada. Soil Science 5: 349.

Kraus, J.E., Kerbauy, G.B., Monteiro, W.R. 2004. Aspectos histoquímicos da formação de protocormóides em ápices radiculares de Catasetum pileatum cultivados in vitro. In: F. Barros \& G.B. Kerbauy (eds.). Orquidologia Sul Americana: uma compilação científica. Secretaria do Meio Ambiente do Estado de São Paulo, São Paulo, pp. 85-89.

Lima, V.F.G.A.P., Menezes, N.L. 2008. Endodermis with meristematic activity in roots of species of Scleria Berg. (Cyperaceae). Boletim de Botânica da Universidade de São Paulo 26 (1): 1-6.

Lynch, J.P., Brown, K.M. 1998. Regulation of root architecture by phosphorus availability. In: J.P. Lynch, J. Deikman (eds.). Phosphorus in plant biology: regulatory roles in molecular, cellular, organismal and ecosystem processes. American Society of Plant Biologists, Rockville, pp. 148-156

Lynch, P. 2018. Rightsizing root phenotypes for drought resistance. Journal of Experimental Botany 69 (13): 3279-3292.

Mani, P.A. 1962. Air-space tissue in Cyperus. Science and Culture 28: 39-40.

Menezes, N.L., Silva, D.C., Arruda, R.C., Melo-de-Pina, G.F., Cardoso, V.A., Castro, N.M., Scatena, V.L., Scremin-Dias, E. 2005. Meristematic activity of the Endodermis and the pericycle in the primary thickening in monocotyledons: considerations on the "PTM". Anais da Academia Brasileira de Ciências 77: 259-274.

Menezes, N.L., Elbl, P.M., Cury, G., Appezzato-daGlória, B., Sasaki, K.L.M., Silva, C.G., Costa, G.R., Lima, V.G.A. 2012. The meristematic activity of the endodermis and the pericycle and its role in the primary thickening of stems in monocotyledonous plants. Plant Ecology \& Diversity 5(2): 153-165.

Munuswamy, E., Amerjothy, S. 2016. Occurrence, type and location of calcium oxalate crystals in selected medicinal plants. Journal of Applied and Advanced Research 1(4): 21-24.

Nakata, P.A. 2003. Advances in our understanding of calcium oxalate crystal formation and function in plants. Plant Science 164 (6): 901-909.

O'Brien, T.P., Feder, N., McCully. M.E. 1965. Polychromatic staining of plant cell walls by toluidine blue O. Protoplasma 59: 368-73.

Paiva, E.A.S. 2019. Are calcium oxalate crystals a dynamic calcium store in plants? New Phytologist 223: 17071711. 
Peterson, R.L. 1992. Adaptations of root structure in relation to biotic and abiotic factors. Canadian Journal of Botany 70 (4): 659-660.

Pita, P.B., Menezes, N.L. 2002. Anatomia da raiz de espécies de Dyckia Schult. F. e Encholirium Mart. ex Schult \& Schult. F. (Bromeliaceae, Pitcairnioideae) da Serra do Cipó (Minas Gerais, Brasil), com especial referência ao velame. Revista Brasileira de Botânica 25: 25-34.

Postma, J.A., Lynch, J.P. 1992. Adaptations of root structure in relation to biotic and abiotic factors. Canadian Journal of Botany 70 (107): 829-841.

Ragni, L., Grebb, T. 2018. Secondary growth as a determinant of plant shape and form. Seminars in Cell \& Developmental Biology 79: 58-67.

Raman, V., Galal, A.M., Avula, B., Sagi, S., Smillie, T.J., Khan, I.A. 2014. Application of anatomy and HPTLC in characterizing species of Dioscorea (Dioscoreaceae). Journal of Natural Medicines 68: 686-698.

Riopel, J.L., Steeves, T.A. 1964. Studies on the roots of Musa acuminatacv. Gros Michel. I. The anatomy and development of main roots. Annals of Botany 28: 475-490.

Rodrigues, A.C., Estelita, M.E.M. 2004. Anatomia da raiz de Cyperus giganteus Vahl (Cyperaceae) em desenvolvimento. Revista Brasileira de Botânica 27: 629-638.

Scatena, V.L., Menezes, N.L. 1996. Anatomia de raízes de Syngonanthus Ruhl. (Eriocaulaceae). Revista Brasileira de Biologia 56: 333-343.

Scatena, V.L., Scremin-Dias, E. 2012. Parênquima, Colênquima e Esclerênquima. In: Appezzato-da-Glória, B. \& Carmello-Guerreiro, S.M. (eds.). Anatomia Vegetal. Editora UFV, Viçosa. pp. 109-127.

Seago, J.L., Marsh, L.C., Stevens, K.J., Soukup, A., Votrubuva, O., Enstone, D.E. 2005. A Re-examination of the root cortex in wetland flowering plants with respect to aerenchyma. Annals of Botany 96: 565-579.

Seo, D.H., Seomun, S., Choi, Y.D., Jang, G. 2020. Root Development and Stress Tolerance in rice: The Key to Improving Stress Tolerance without Yield Penalties. International Journal of Molecular Science 21: 1-13.

Seubert, E. 1997. Root anatomy of palms. Flora 192: 81-103.

Souza, V.C. \& Lorenzi, H. 2012. Botânica sistemática: guia ilustrado para identificação das famílias de Fanerógamas nativas e exóticas no Brasil, baseado em APG III. 3.ed. Instituto Plantarum, Nova Odessa.
Stone, B.C. 1972. A Reconsideration of the Evolutionary Status of the Family Pandanaceae and its Significance in Monocotyledon Phylogeny. The Quarterly Review of Biology 47(1).

Stone, B.C. 1982. New Guinea Pandanaceae: first approach to ecology and biogeography Benjamin C. Stone Monographiae Biologicae. Dr W. Junk Publishers, Londres.

Tomescu, A.M.F., Groover, A.T. 2019. Mosaic modularity: an updated perspective and research agenda for the evolution of vascular cambial growth. New Phytologist 222: 1719-1735.

Tomlinson, P.B. 1969. On the morphology and anatomy of turtle grass, Thalassia testudinum (Hydrocharitaceae). II. Anatomy and development of the root in relationt of unction. Bulletin of Marine Science 19: 57-71.

Tomlinson, P.B., Zimmermann, P.H. 1969. Vascular anatomy of monocotyledons with secondary growth - an introduction. Journal of the Arnold Arboretum 50 (2): 159-179.

Torres-Martínez, H.H., Rodríguez-Alonso, G., Shishkova, S., Dubrovsky, J.G. 2019. Lateral root primordium morphogenesis in angiosperms. Frontiers in Plant Science 10: 1-19.

Van Fleet, D.S. 1961. Histochemistry and function of the endodermis. The Botanical Review 27: 165-220.

Vianna, W.O., Soares, M.K.M., Appezzato-da-Glória, B. 2001. Anatomia da raiz escora de Philodendron bipinnatifidum Schott (Araceae). Acta Botanica Brasilica 15: 313-320.

Wilder, G.J. 1986. Anatomy of first-order roots in the Cyclantaceae (Monocotiledonea). I. Epidermis, cortex and pericycle. Canadian Journal of Botany 64: 26222644.

Xiao, T.T., van Velzen, R., Kulikova, O., Franken, C., Bisseling, T. 2019. Lateral root formation involving cell division in both pericycle, cortex and endodermis is a common and ancestral trait in seed plants. Development 146: $1-5$.

Zhu, J., Brown, K.M., Lynch, J.P. 2010. Root cortical aerenchyma improves the drought tolerance of maize (Zea mays L.). Plant, Cell \& Environment 33: 740-749.

Recebido: 25.09.2020 Aceito: 03.03.2021

Editor Associado: Juliana Hanna Leite El Ottra 